\title{
Electromagnetically induced transparency in bulk and microcavity semiconductors
}

\author{
F. Bassani ${ }^{\mathrm{a}}$, G.C. La Rocca ${ }^{\mathrm{a}, *}$, M. Artoni ${ }^{\mathrm{b}}$ \\ ${ }^{a}$ Scuola Normale Superiore, Piazza dei Cavalieri 7, 56126 Pisa, Italy \\ ${ }^{\mathrm{b}}$ Dipartimento di Chimica e Fisica per l'Ingegneria e per i Materiali, Università di Brescia, Via Valotti 9, 25133 Brescia, Italy
}

Available online 21 September 2004

\begin{abstract}
We discuss our recent results on electromagnetically induced transparency (EIT) effects based on intrinsic free exciton and biexciton states in semiconductors. The $\Lambda$ configuration obtained from the $1 \mathrm{~S}$ and $2 \mathrm{P}$ yellow exciton levels of $\mathrm{Cu}_{2} \mathrm{O}$ leads to a well-developed EIT regime, akin to the atomic case. The coherent driving of the exciton-biexciton transition in $\mathrm{CuCl}$ induces a tunable transparency window within the polaritonic stop-band, due to the presence of a third polariton branch in the dressed system. In a microcavity configuration, this gives rise to three reflectivity dips in the strong coupling regime.
\end{abstract}

(C) 2004 Elsevier B.V. All rights reserved.

PACS: 71.35.-y; 71.36. $+\mathrm{c} ; 78.20 .-\mathrm{e}$

Keywords: EIT; Exciton; Biexciton; Polariton; $\mathrm{Cu}_{2} \mathrm{O} ; \mathrm{CuCl}$

\section{Introduction}

There has been over the past decade a flurry of interest in techniques that enable one to control effects of quantum coherence in three-level atomic systems. Early experiments have been carried out by Gozzini's group in Pisa, where a peculiar quantum coherence effect in optical pumping experiments with sodium atoms was first observed,

\footnotetext{
*Corresponding author. Tel.: + 39-050-509111; fax: + 39050-563513.

E-mail address: larocca@sns.it (G.C. La Rocca).
}

and later by Stroud's group in Rochester. In Gozzini's experiment, for instance, when the separation between two Zeeman sublevels of the $3 \mathrm{~S}$ ground state happened to be equal to the frequency difference between two nearly resonant laser beams both coupled to the 3P excited level of the sodium $\mathrm{D}_{1}$ line, no absorption took place and hence no fluorescence off the excited state was observed. The sodium atoms behaved as though they were transparent to the incident resonant light and the phenomenon was originally referred to as the "riga nera" (black line) effect. Quantum coherence involving the $3 \mathrm{~S}$ ground state doublet, 
pumped into a coherent superposition state where the population is trapped due to destructive interference between the two distinct absorption paths to the excited 3P level, was the key to interpret such an intriguing effect, as suggested by Arimondo and coworkers. Over the past decade and long after Gozzini's pioneering work [1], coherent population trapping in atoms has certainly revived the interest of the scientific community as it lay at the heart of important phenomena such as electromagnetically induced transparency (EIT), subrecoil laser cooling, lasing without inversion, adiabatic transfer and slow light, just to mention a few [2].

For many potential applications solid state media are preferred. In such media, however, decoherence takes place rather quickly which makes effects of EIT rather difficult to observe. EIT in solids has been so far attained in a class of materials exhibiting defect states such as, e.g., rareearth impurities [3] or color center states [4]. EIT effects have also been observed in semiconductor quantum wells [5]. In both cases, inhomogeneous broadening plays a very significant role. On a whole different ground, however, EIT schemes could be implemented in semiconductors exhibiting narrow intrinsic resonances associated with delocalized free exciton and biexciton levels. In particular, it has been recently anticipated that forbidden yellow exciton states of $\mathrm{Cu}_{2} \mathrm{O}$ [6] and exciton and biexciton states in $\mathrm{CuCl}$ [7] can lead to large EIT effects. These recent developments are illustrated here, including the possibility of combining EIT effects with photon confinement in a microcavity configuration.

\section{A $\Lambda$ EIT configuration based on the yellow exciton of $\mathrm{Cu}_{2} \mathrm{O}$}

Because conduction and valence bands in cuprous oxide $\left(\mathrm{Cu}_{2} \mathrm{O}\right)$ both have even parity, the transition from the ground to the $1 \mathrm{~S}$ exciton level is forbidden, whereas the one to the $2 \mathrm{P}$ exciton level is allowed but rather weak. The $1 \mathrm{~S}-2 \mathrm{P}$ transition is also allowed. The ground, $1 \mathrm{~S}$ and $2 \mathrm{P}$ exciton levels, represented in the inset of Fig. 1, respectively, by the states $|0>| 1>$, and $\mid 2>$, give

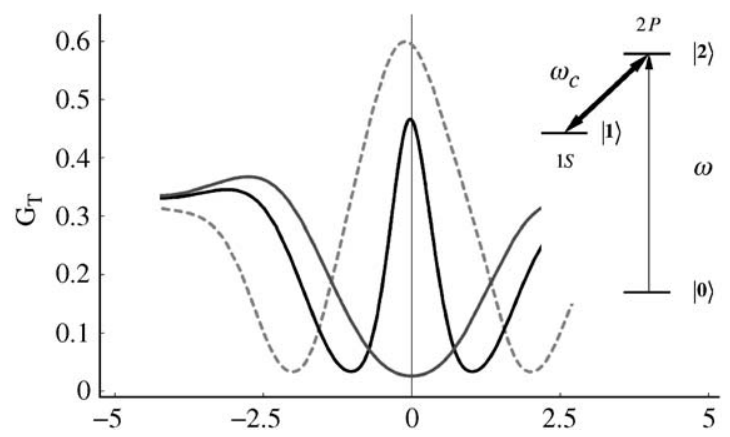

Fig. 1. Transmission coefficient $G_{\mathrm{T}}$ vs. the probe frequency detuning in units of $\gamma_{2 \mathrm{P}}$; the background dielectric constant is $\varepsilon_{\infty}=6.5+\mathrm{i} 2 \times 10^{-3}$. The coupling Rabi frequencies are $\Omega_{\mathrm{c}} / \gamma_{2 \mathrm{P}}=2$ (black solid) and 4 (gray dash) while in the absence of the coupling beam $\left(\Omega_{\mathrm{c}}=0\right)$ the transmission is described by the solid gray curve.

rise to a typical $\Lambda$ configuration where EIT could be implemented. In particular, we are interested in the optical response experienced by a weak probe beam of frequency $\omega$ tuned around the frequency $\omega_{2 \mathrm{P}}(2.148 \mathrm{eV})$ of the $2 \mathrm{P}$ exciton transition line, while a strong coupling beam of frequency $\omega_{\mathrm{c}}$ and variable Rabi frequency $\Omega_{\mathrm{c}}$ is nearly resonant with the $1 \mathrm{~S}-2 \mathrm{P}$ exciton transition of frequency $\omega_{2 \mathrm{P}-1 \mathrm{~S}}=$ $\omega_{2 \mathrm{P}}-\omega_{1 \mathrm{~S}}(115 \mathrm{meV})$. The optical susceptibility experienced by the probe in the presence of the coupling beam can be evaluated and this is given by Artoni et al. [6]

$\chi_{\mathrm{p}}(\omega)=\frac{A \gamma_{2 \mathrm{P}}\left(\delta_{\mathrm{p}}-\delta_{\mathrm{c}}-\mathrm{i} \gamma_{1 \mathrm{~S}}\right)}{\left(\delta_{\mathrm{p}}-\mathrm{i} \gamma_{2 \mathrm{P}}\right)\left(\delta_{\mathrm{p}}-\delta_{\mathrm{c}}-\mathrm{i} \gamma_{1 \mathrm{~S}}\right)-\Omega_{\mathrm{c}}^{2} / 4}$,

where $\delta_{\mathrm{p}}=\omega_{2 \mathrm{P}}-\omega$ and $\delta_{\mathrm{c}}=\omega_{2 \mathrm{P}-1 \mathrm{~S}}-\omega_{\mathrm{c}}$ are the two relevant detunings and where the factor $A \simeq$ 0.02 is proportional to the $2 \mathrm{P}$ exciton oscillator strength. All relevant spectroscopic parameters for the "yellow exciton series" in $\mathrm{Cu}_{2} \mathrm{O}$ are available [8], the 1S state linewidth $\hbar \gamma_{1 \mathrm{~S}} \simeq 0.1 \mathrm{meV}$ is small compared to the 2P linewidth $\hbar \gamma_{2 \mathrm{P}} \simeq 1 \mathrm{meV}$ and a well-developed EIT can be established in the presence of the coupling beam [6]. The above dressed susceptibility $\chi_{\mathrm{p}}(\omega)$ has the same form as that of an atomic three-level system, and because the $2 \mathrm{P}$ exciton is only weakly allowed the value of $A$ is also comparable with that of an ultracold sample of alkali atoms. It is also worth noticing that polaritonic effects are in this case immaterial. 
Fig. 1 shows the probe transmission profile through a $d=35 \mu \mathrm{m}$ thick slab yielding a nearly $50 \%$ transmission in the presence of the coupling beam suggesting quite a large EIT effect. Apart from the background absorption, which is included here, the $1 \mathrm{~S}$ exciton linewidth $\gamma_{1 \mathrm{~S}}$ is the material parameter that mostly limits the possibility of achieving even larger EIT effects in $\mathrm{Cu}_{2} \mathrm{O}$. The experimental observation of a fully developed EIT regime for intrinsic exciton lines in semiconductors would not only open the way to device applications, but also to assess peculiar effects of the electrodynamics of continuous media such as, e.g., in the case of the Fresnel-Fizeau light-drag [9] or in the emission of Cherenkov radiation [10] in the EIT regime.

\section{Exciton-biexciton coupling in $\mathrm{CuCl}$}

Copper chloride $(\mathrm{CuCl})$ is a prototype example of a semiconductor having an allowed interband transition and quite pronounced exciton and biexciton resonances, and it exhibits a fully developed polaritonic stop-band [11]. A pump coherently driving the exciton-biexciton transition allows for a well-developed transparency within the stop-band where a probe pulse may propagate, although with a strongly reduced group velocity [7]. The large oscillator strength of the exciton-biexciton transition and the very narrow linewidth and long coherence time of the biexciton state in the small wave vector region favor quite appreciable degrees of transparency. The phenomenon is reminiscent of EIT effects occurring in three-level atomic systems, but the physics of the induced transparency within an otherwise reflecting stopband relies on a frequency and wave vector selective polaritonic mechanism [12]. The $\mathrm{CuCl}$ response to a weak probe beam of frequency $\omega$ and wave vector $\vec{k}$, in the presence of the strong coupling beam of frequency $\omega_{\mathrm{c}}$ and wave vector $\vec{k}_{\mathrm{c}}=-\vec{k}$, turns out to be described by the following dielectric constant [13]:

$$
\varepsilon(k, \omega)=\varepsilon_{\mathrm{b}}+\frac{\varepsilon_{\mathrm{b}} \Delta_{\mathrm{LT}}}{\hbar \omega_{x}(k)-\hbar \omega-\mathrm{i} \gamma_{x}+\Sigma}
$$

with

$\Sigma=\frac{\beta}{\hbar \omega+\hbar \omega_{\mathrm{c}}-\hbar \omega_{m}\left(k-k_{\mathrm{c}}\right)+\mathrm{i} \gamma_{m}}$.

Here, $\omega_{x}(k)=\omega_{\mathrm{T}}+\hbar k^{2} /\left(2 m_{x}\right)$ and $\omega_{m}(k)=\omega_{\mathrm{M}}+$ $\hbar k^{2} /\left(2 m_{m}\right)$ denote, respectively, the exciton and biexciton dispersions; $\Sigma$ describes the nonlinearity due to the coherent coupling beam with $\beta$ proportional to the pump intensity and to the oscillator strength of the exciton-biexciton transition. The exciton and biexciton energies $\hbar \omega_{\mathrm{T}}=3.202 \mathrm{eV}$ and $\hbar \omega_{\mathrm{M}}=6.372 \mathrm{eV}$ at $k=0$, their masses $m_{x}$ and $m_{m}$ and linewidths $\gamma_{x}=$ $50 \mu \mathrm{eV}$ and $\gamma_{m}=15 \mu \mathrm{eV}$, the background dielectric constant $\varepsilon_{\mathrm{b}}$, and the exciton longitudinal-transverse splitting $\Delta_{\mathrm{LT}}=5.65 \mathrm{meV}$ are all known parameters from experiments.

Even though the form of the dressed dielectric constant above corresponds to that of a three-level atom, in the present case the oscillator strength is large and $\mathrm{CuCl}$, unlike $\mathrm{Cu}_{2} \mathrm{O}$ or atoms, shows pronounced polaritonic effects. In the absence of the exciton-biexciton coherent coupling, i.e. when the pump is off $(\beta \rightarrow 0)$, one obtains from Maxwell's equations the usual upper and lower polariton dispersion branches and a polaritonic stop-band within which the probe is nearly completely reflected. Conversely, the exciton-biexciton coherent coupling induced by the pump leads to three solutions of the probe dispersion equation $\left(c^{2} k^{2} / \omega^{2}\right)=\varepsilon(k, \omega)$. The third polariton branch appears in the frequency region of the exciton resonance where the probe reflectivity is expected to be affected [14]. Fig. 2 shows the probe normal incidence transmission spectra through a thin $\mathrm{CuCl}$ slab with and without the coupling beam. A pronounced transparency window in correspondence with the pump-induced dispersion branch opens up within the polaritonic frequency stopband around $\omega \simeq \omega_{m}\left(k-k_{\mathrm{c}}\right)-\omega_{\mathrm{c}}$. It is worthwhile noting that transparency crucially depends on the biexciton linewidth at a small wave vector $\gamma_{m}$, yet much less on the exciton linewidth $\gamma_{x}$. The transparency window can be readily controlled by changing the pump frequency over a rather wide spectral range of several $\mathrm{meV}$ which corresponds to the entire polaritonic stop-band, though absorption here is still quite significant. 

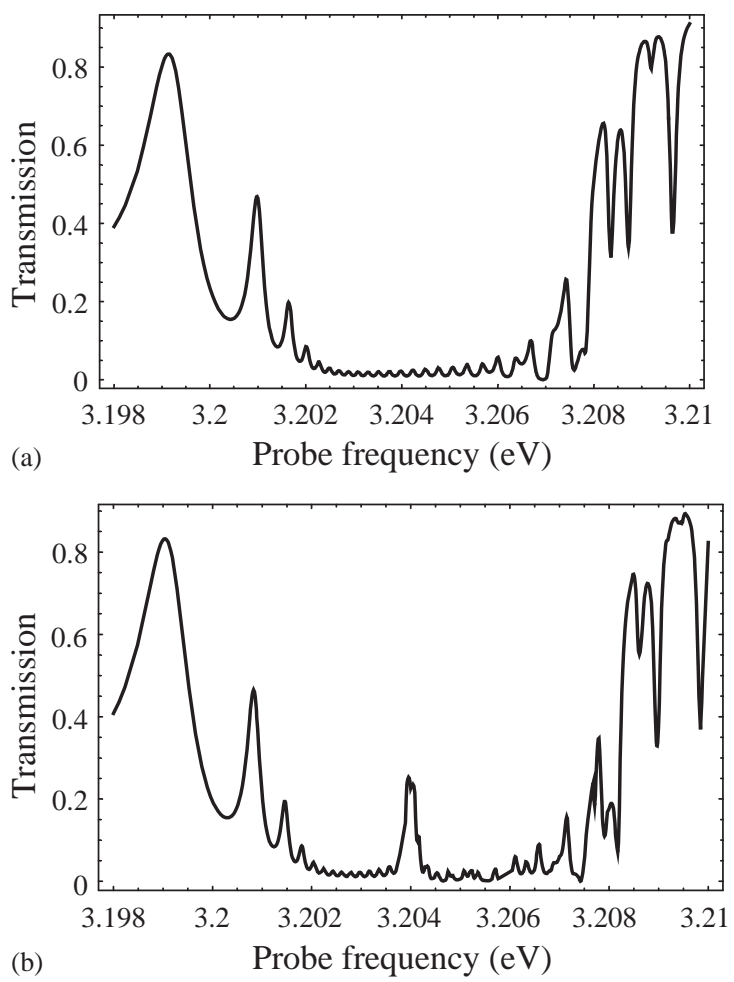

Fig. 2. Probe transmission spectra through a $0.15 \mu \mathrm{m}$ thick $\mathrm{CuCl}$ film: (a) no pump, (b) with a pump of frequency $\hbar \omega_{\mathrm{c}}=$ $3.168 \mathrm{meV}$ and intensity corresponding to $\beta=5 \times 10^{-7} \mathrm{eV}^{2}$.

\section{EIT in a semiconductor microcavity}

Effects associated with biexciton resonances in strongly coupled semiconductor microcavities are an area of current interest both theoretically and experimentally [15]. Even in microcavities, the exciton-biexciton coherent coupling is expected to originate a third cavity polariton branch besides the usual upper and lower ones separated by the Rabi splitting. Using a material with well developed and strongly bound exciton and biexciton resonances such as $\mathrm{CuCl}$ would make the coherent coupling effects quite visible. No experimental results, to the best of our knowledge, have been reported on $\mathrm{CuCl}$ based microcavities. In the following, we use model parameters extrapolated from the bulk values to examine the behavior of a microcavity containing a $\mathrm{CuCl}$ quantum well. Such an extrapolation procedure should be in good order at least from a qualitative point of view. Specifically, we take a Rabi splitting $\Delta_{\mathrm{R}}$ of about $50 \mathrm{meV}$, the two-dimensional biexciton binding energy of about $100 \mathrm{meV}$ and the twodimensional exciton and biexciton broadenings of the order of $1 \mathrm{meV}$, allowing for interface roughness scattering. The coupling $\beta \approx 10^{-4} \mathrm{eV}^{2}$ is taken to be about 100 times larger than that used for the free standing slab above; such a choice takes into account the enhancement of the oscillator strength of the exciton-biexciton transition due to the 2D confinement and, most importantly, the resonant effect of the cavity on the pump electric field. Thus, the actual incident pump power need not be higher than in the previous case. The microcavity probe transmission and reflection spectra are obtained by using a transfer matrix method whereby the $\mathrm{CuCl}$ quantum well is included via a local dressed dielectric function of the same form as above. The cavity is tuned below the exciton resonance with a detuning close to the biexciton binding energy. The exciton-biexciton quantum coherence can then be driven by a resonant pump entering the cavity at near normal incidence.

The microcavity reflectivity dips are shown in Fig. $3 \mathrm{a}$ as a function of probe frequency and parallel wave vector $q$ : it is clear the presence of the third cavity polariton branch is induced by the pump. The corresponding section for $q$ close to the bare exciton-cavity mode anticrossing is shown in Fig. 4a, while Fig. 4b shows the corresponding absorption spectrum. Fig. 5 shows the same plots at normal incidence $(q=0)$. When compared with the case of the free standing slab, it has to be noticed that much larger biexciton (and exciton) broadenings do not prevent the observation of the pump induced transparency associated to the third cavity polariton branch. It is worthwhile to notice, in addition, that the exciton-biexciton coherent coupling effects here considered are of the same nature as those described by the "average polarization model" of Ref. [16], yet quite distinct from effects related to the presence of an incoherent exciton population that would transfer oscillation strength to the biexciton transition [17]. In particular, as shown in Fig. 3c, a very good approximation to the dispersion $\omega(k)$ of the three cavity polariton modes of the coherently dressed system can be obtained from the following 

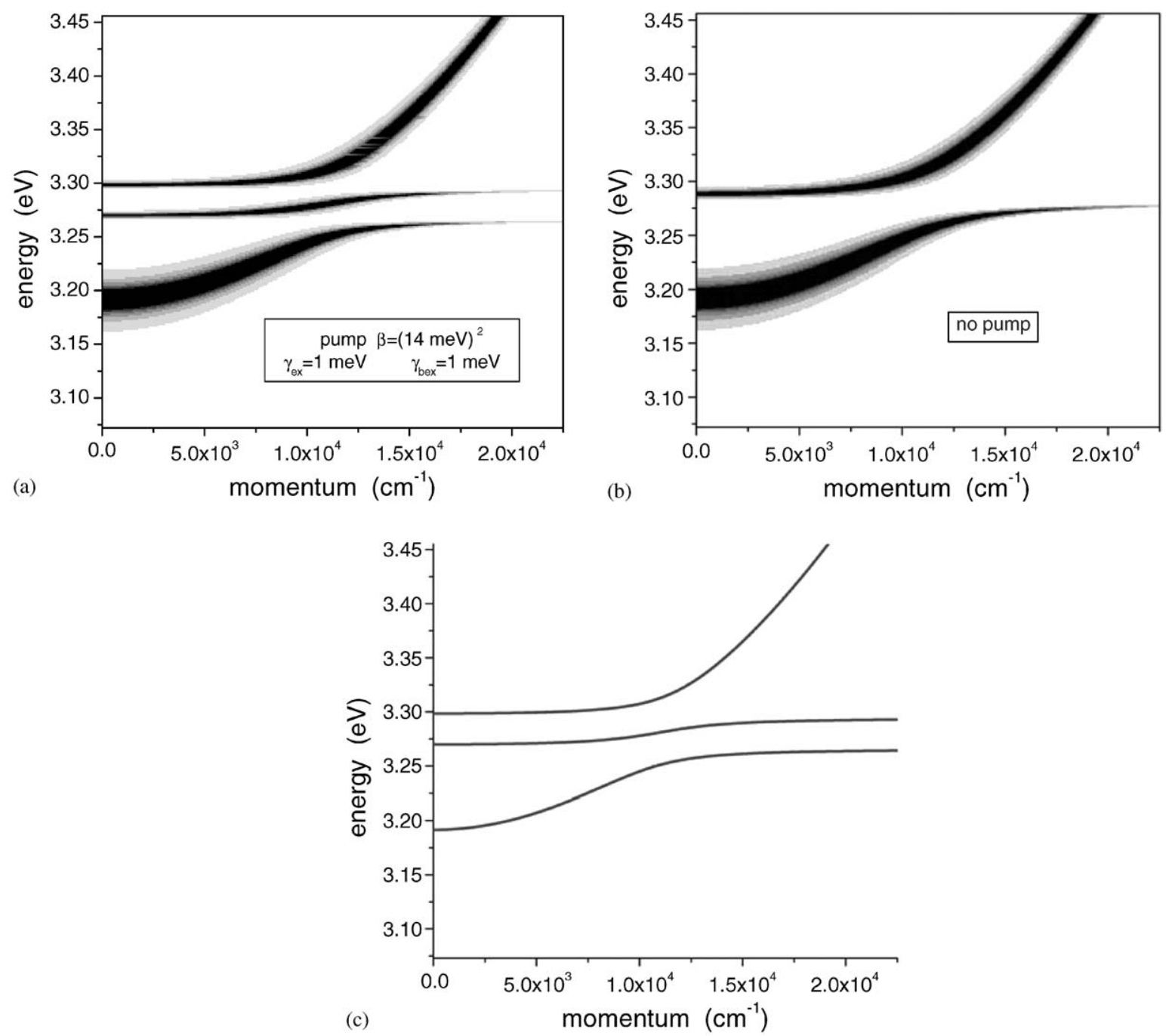

Fig. 3. Micro-cavity reflectivity dips as a function of probe frequency and parallel wave vector with (a) and without (b) the pump beam; (c) shows the dispersion of the three polariton modes of the dressed system from the coupled oscillator model (see text) corresponding to (a).

determinantal equation of a coupled oscillator model:

$$
\begin{aligned}
& \operatorname{det}\left(\begin{array}{ccc}
\hbar\left(\omega_{\mathrm{MC}}-\omega\right) & \Delta_{\mathrm{R}} / 2 & 0 \\
\Delta_{\mathrm{R}} / 2 & \hbar\left(\omega_{x}-\omega\right) & \sqrt{\beta} \\
0 & \sqrt{\beta} & \hbar\left(\omega_{m}-\omega_{\mathrm{c}}-\omega\right)
\end{array}\right) \\
& =0,
\end{aligned}
$$

where $\omega_{\mathrm{MC}}(k)$ is the empty cavity photon mode and the three states corresponding to the rows of the above matrix from top to bottom are: (i) one cavity photon, no exciton, no biexciton, $N$ photons in the pump beam $(N \gg 1)$, (ii) no cavity photon, one exciton, no biexciton, $N$ photons in the pump beam, (iii) no cavity photon, no exciton, one biexciton, $(N-1)$ photons in the pump beam.

\section{Future directions}

Apart from the microcavity configuration considered above, a promising way to combine EIT 


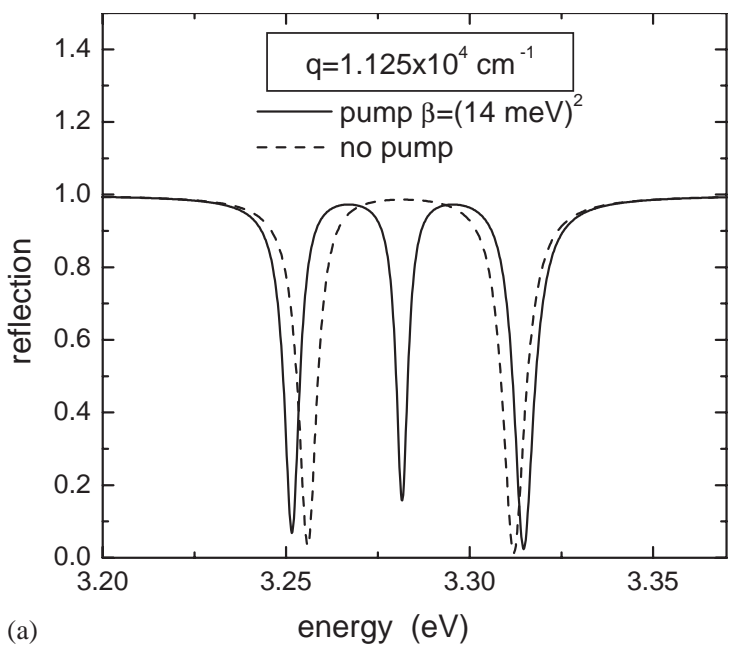

(a)

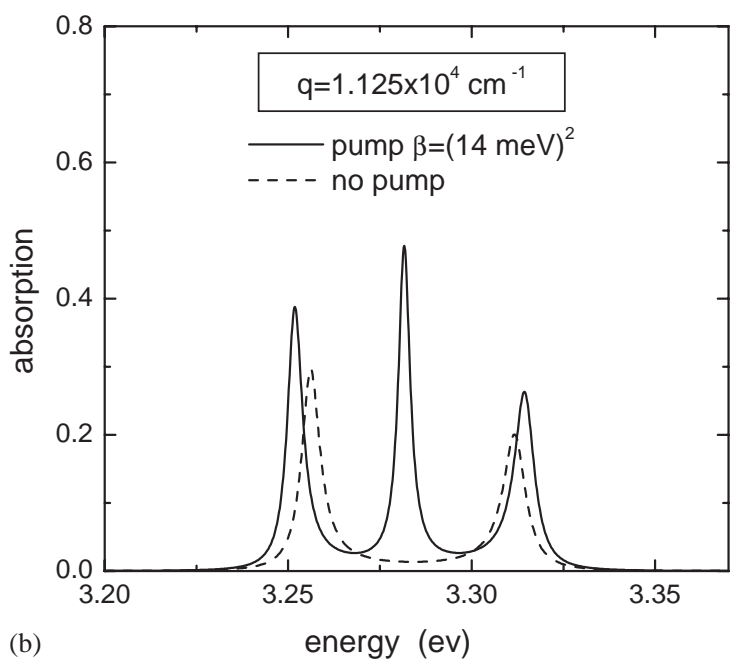

Fig. 4. Reflectivity (a) and absorption (b) spectra near the anticrossing (parameters as in Fig. 3).

and photon confinement would be through the realization of a coherently controlled photonic band gap system, as recently considered in atoms [18]. Photonic band gaps appear in a medium having a response periodically varying in space on the scale of an optical wavelength, which is usually realized at the growth by using materials with different dielectric constants. A periodic modulation of the response can, however, be obtained through EIT using a standing wave configuration for the pump (rather than a travelling wave) as in this case the coupling beam Rabi frequency would
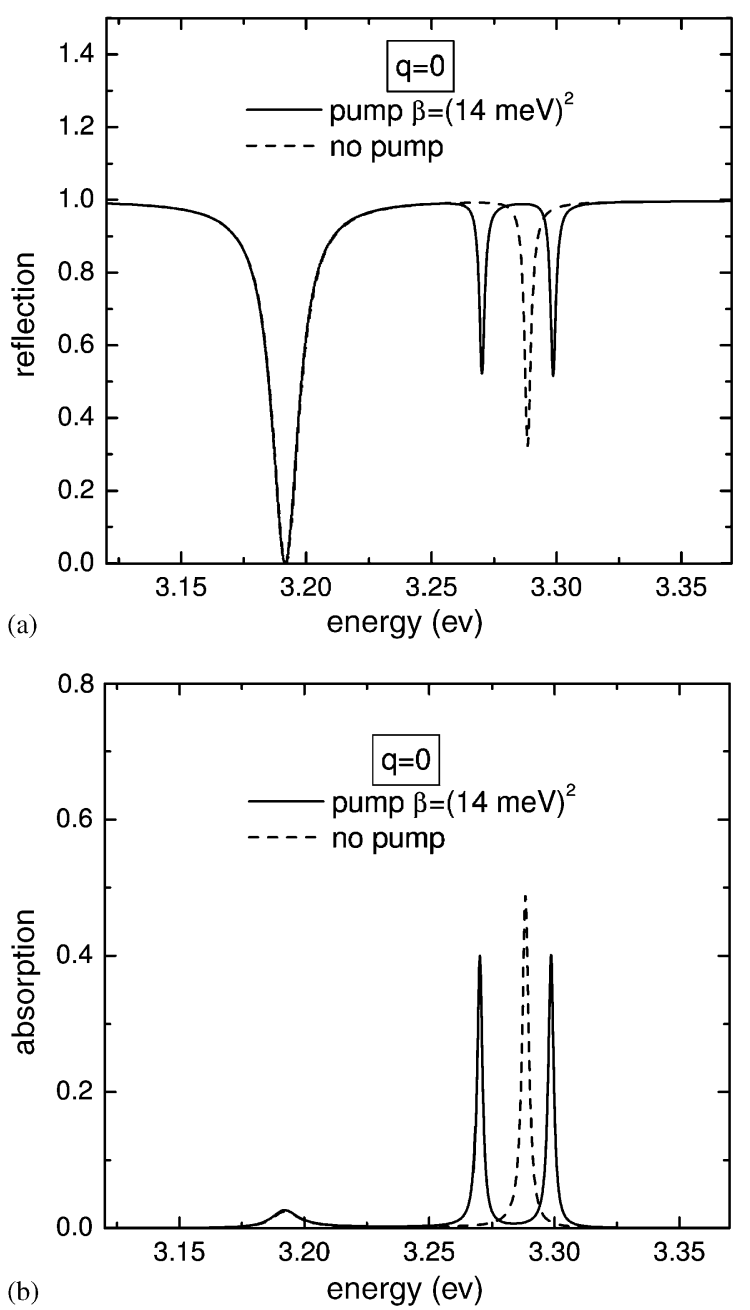

Fig. 5. Reflectivity (a) and absorption (b) spectra at normal incidence (parameters as in Fig. 3).

vary periodically in space. This would allow the realization of an all optical tunable photonic crystal and opens the way to a host of applications, particularly in optical computing and quantum information processing and storage.

\section{Dedication and acknowledgements}

We are very happy to present this contribution on electromagnetically induced transparency in semiconductors to the Festschrift honoring Professor Vladimir Moiseevich Agranovich on his 
75th anniversary. Semiconductor optics is a subject to which he has contributed with so many fundamental results and a subject in which he has helped us in so many ways; not only with his deep mastery of all aspects of electrodynamics of continuous media and light-matter interaction, but also with his enduring enthusiasm and friendly encouragement. Thank you very much, Volodya! We wish you continued excellent health and intellectual vigor, and we look forward to your future contributions to our common fields of interest for a long time to come.

It is a pleasure to thank the many colleagues who have collaborated with us on EIT-related problems, and particularly D. Balagurov, I. Carusotto, F. Cataliotti, S. Chesi, M. Inguscio and A. Mysyrowicz. We ackwowledge financial support from MIUR (Grant PRIN 2002028850 and Actione Integrada Italia-Spagna) and the EC (HYTEC contract HPRN-CT-2002-00315).

\section{References}

[1] G. Alzetta, A. Gozzini, L. Moi, G. Orriols, Nuovo Cimento 36 (1976) 5;

E. Arimondo, G. Orriols, Lett. Nuovo Cimento 17 (1976) 333.

[2] S. Harris, Physics Today 50 (1997) 36;

E. Arimondo, in: E. Wolf (Ed.), Progress in Optics XXXV, Elsevier Science, Amsterdam, 1996, p. 257;

A.B. Matsko, O. Kocharovskaya, Y. Rostovtsev, G.R. Welch, A.S. Zibrov, M.A. Scully, Adv. At. Mol. Opt. Phys. 46 (2001) 191.

[3] B.S. Ham, M.S. Shahriar, R.P. Hemmer, Opt. Lett. 22 (1997) 1138;

K. Ichimura, K. Yamamoto, N. Gemma, Phys. Rev. A 58 (1998) 4116;

A.V. Turukhin, V.S. Sudarshanam, M.S. Shahriar, J.A. Musser, B.S. Ham, P.R. Hemmer, Phys. Rev. Lett. 88 (2002) 23602.

[4] C. Wei, N.B. Manson, Phys. Rev. A 60 (1999) 2540.
[5] G.B. Serapiglia, E. Parpalakis, C. Sirtori, K.L. Vodopyanov, C.G. Phillips, Phys. Rev. Lett. 84 (2000) 1019; M.C. Phillips, H. Wang, I. Rumyantsev, N.H. Kwong, R. Takayama, R. Binder, Phys. Rev. Lett. 91 (2003) 183602.

[6] M. Artoni, G.C. La Rocca, F. Bassani, EuroPhys. Lett. 49 (2000) 445;

M. Artoni, G.C. La Rocca, I. Carusotto, F. Bassani, Phys. Rev. B 65 (2002) 235422.

[7] S. Chesi, M. Artoni, G.C. La Rocca, F. Bassani, A. Mysyrowicz, Phys. Rev. Lett. 91 (2003) 57402.

[8] S. Nikitine, J.B. Grun, M. Sieskind, J. Phys. Chem. Solids 17 (1961) 292;

D. Fröhlich, A. Nothe, K. Reimann, Phys. Rev. Lett. 55 (1985) 1335;

D. Fröhlich, Ch. Neumann, B. Uebbing, R. Wille, Phys. Status Solidi (b) 159 (1990) 297.

[9] M. Artoni, I. Carusotto, G.C. La Rocca, F. Bassani, Phys. Rev. Lett. 86 (2001) 2549;

I. Carusotto, M. Artoni, G.C. La Rocca, F. Bassani, Phys. Rev. A 68 (2003) 63819.

[10] I. Carusotto, M. Artoni, G.C. La Rocca, F. Bassani, Phys. Rev. Lett. 87 (2001) 64801;

M. Artoni, I. Carusotto, G.C. La Rocca, F. Bassani, Phys. Rev. E 67 (2003) 46609.

[11] V.M. Agranovich, V.L. Ginzburg, Crystal Optics with Spatial Dispersion and Excitons, Springer, Berlin, 1984.

[12] R. Shimano, M. Kuwata-Gonokami, Phys. Rev. Lett. 72 (1994) 530.

[13] R. März, S. Schmitt-Rink, H. Haug, Z. Phys. B 40 (1980) 9;

K. Cho, J. Phys. Soc. Japan 54 (1985) 4444.

[14] V. May, K. Henneberger, F. Henneberger, Phys. Status Solidi (b) 94 (1979) 611;

E. Hanamura, Phys. Rev. B 44 (1991) 8514; A.L. Ivanov, H. Haug, L.V. Keldysh, Phys. Rep. 296 (1998) 237.

[15] T. Baars, G. Dasbach, M. Bayer, A. Forchel, Phys. Rev. B 63 (2001) 165311 and references therein.

[16] U. Neukirch, S.R. Bolton, N.A. Fromer, L.J. Sham, D.S. Chemla, Phys. Rev. Lett. 84 (2000) 2215.

[17] M. Saba, F. Quochi, C. Ciuti, U. Oesterle, J.L. Staehli, B. Deveaud, G. Bongiovanni, A. Mura, Phys. Rev. Lett. 85 (2000) 385.

[18] A. Andre, M.D. Lukin, Phys. Rev. Lett. 89 (2002) 143602; M. Bajcsy, A.S. Zibrov, M.D. Lukin, Nature 426 (2003) 638. 